\title{
Tide-Induced Eddies and Upwelling in a Semi-enclosed Basin: Nan Wan
}

\author{
H.-J. Lee ${ }^{a}$, S.-Y. Chao ${ }^{b}$, K.-L. Fan ${ }^{a}$ and T.-Y. Kuo ${ }^{a}$ \\ ${ }^{a}$ Institute of Oceanography, National Taiwan University, Taipei, Taiwan, R.O.C. \\ ${ }^{b}$ Horn Point Laboratory, University of Maryland Center for Environmental Science, Cambridge, \\ Maryland 21613-0775, U.S.A.
}

Received 8 February 1999 and accepted in revised form 21 fune 1999

\begin{abstract}
Nan Wan is a $20 \mathrm{~km}$ wide tidally-dominated embayment situated between two headlands on the south coast of Taiwan. During spring tides, sudden sea-surface temperature drops occur twice each tidal cycle in the western and central regions of the bay, but only once in the eastern region. Shipboard ADCP surveys, moored measurements and numerical modelling results demonstrate that the headlands on either side of the bay generate strong tidally-induced eddies within the bay on each phase of the tide. The geometry of the region leads to considerable difference in size between the flood and ebb eddies. The flood eddy fills the entire basin, while the ebb eddy fills the western and central region only. The strong (relative vorticity $\approx 10-16 f$ ) cyclostrophic eddies are only weakly affected by Earth's rotation, and thus upwelling occurs within each eddy, causing two temperature drops per tidal cycle in the western and central region, while only one drop in the eastern region.

(C) 1999 Academic Press
\end{abstract}

Keywords: tides; numerical models; upwelling; embayment; eddies; Taiwan Coast

\section{Introduction}

The southernmost embayment of Taiwan, locally known as Nan Wan (Figure 1), is a semi-enclosed basin bounded zonally by two capes. Distance between the two capes is about $14 \mathrm{~km}$. The east cape, locally known as O-Luan-Bi (OLB), protrudes farther south than does the west cape, locally known as Mou-Bi-Tou (MBT). The semi-enclosed basin borders the Pacific Oceans to the east and Taiwan Strait to the west. A zonally elongated seamount partially blocks the bay mouth; maximum height of the seamount reaches about $50 \mathrm{~m}$ below the sea surface. There is practically no continental shelf on the west side of the Bay. On the east side, the shallow continental shelf shoreward of $80 \mathrm{~m}$ isobath is about $4 \mathrm{~km}$ wide, with isobaths running more or less parallel to the coastline. Between the seamount to the south and land masses to the north, the deeper portion of Nan Wan forms an arc-shaped channel open at both ends.

Tidal currents dominate the circulation in Nan Wan and vicinity, often exceeding $2 \mathrm{~ms}^{-1}$ in strength during the spring tides (Lee et al., 1997). Monsoonal winds over Nan Wan are from the north-east from late autumn to early spring, and from south-west in summer. Except for a few typhoon events in the autumn, wind-driven motions are normally much weaker than the prevailing tidal currents. Beyond wind-induced currents, past point measurements indicate that the remotely forced circulation of large scale origin is even weaker. The highly repetitive nature of tides allows for synthesis of physical processes based on observations from different periods. In fact, much of our understanding about tidally induced upwelling or cold water intrusions in Nan Wan is built on a series of processoriented observational efforts not overlapping in time.

Diurnal and semi-diurnal tides in Nan Wan are about equal in amplitude (Lee et al., 1997); both are modulated by a pronounced spring-neap cycle. Around neaps, sudden temperature drops, if any, are from faint to nonexistent. During spring tides, tidally induced upwelling often causes sudden temperature drops during each tidal cycle in Nan Wan, lasting several hours each. When conditions are favourable, the temperature drops can be as large as $10^{\circ} \mathrm{C}$ in some regions. For example, persistent north-east monsoon in winter months often raises the thermocline in the bay, allowing tide-induced upwelling to trigger large temperature drops. In one widely publicized example on 24 November, 1988, a cold event decreased the sea surface temperature to about $14{ }^{\circ} \mathrm{C}$ in a few hours, resulting in mass kill of fishes. The incidence provided the impetus to launch the decadelong observational and numerical modelling efforts. 


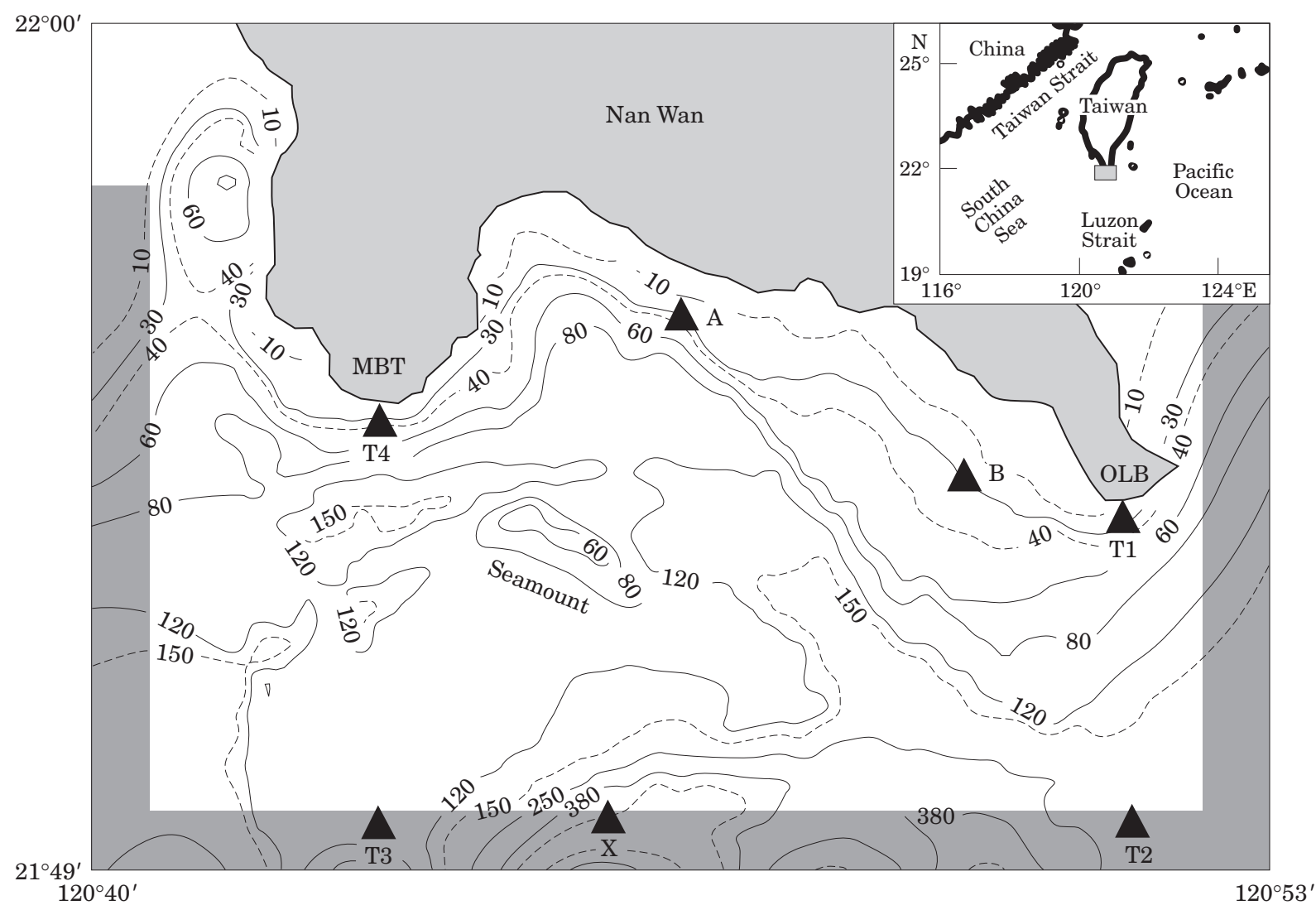

FIGURE 1. Large scale setting, model domain, temperature stations (A, B, and X) and tide gauge stations (T1, T2, T3 and T4). Isobaths are in metres. Shading indicates sponge layer areas adjacent to open boundaries. Northern open oceans to the east and west of the Bay are truncated by zonal walls placed at the northern extremes of the sponge layer areas.

This paper first summarizes recent observations and previous modelling results. A numerical tidal model is then presented to replicate salient features revealed from recent observations. Mechanisms leading to the tidally induced upwelling are identified, in the hope that they would help interpret like phenomena in semi-enclosed basins elsewhere.

\section{Recent observations}

In October-December 1995, 12 temperature sensors, four current meters and six tide gauges were strategically deployed in and around Nan Wan to better identify the cold water intrusion process. Details were summarized in Lee et al. (1997). It was determined that the phenomenon of sudden temperature drops during spring tides is confined mostly to within the bay and decays farther out to the sea. Further, characteristics of sudden temperature drops in the central and western reaches differ from that on the east side of the Bay. The sudden temperature drop occurs twice per day in the central and west sides of the basin, but only once per day on the east side. To quantify further, Figure 2 shows time series of temperature at stations $\mathrm{A}$ and $\mathrm{B}$, and sea level at OLB on 12 November, 1995. Station locations are given in Figure 1. This is a typical spring period. Temperature sensors were placed at $15 \mathrm{~m}$ depth. Station A is typical for the central and western reaches of the basin; sudden temperature drops occur twice daily. The first drop occurs shortly after the lower low-water as the tide is approaching lower high-water. The second drop occurs as the tide is turning from lower highwater to higher low-water. Subsequent relaxation of the second temperature drop occurs as the tide approaches higher high-water. Station B is over the continental shelf on each side of the basin. The sudden temperature drop is once per day at station $B$, occurring between the two temperature drops at station A.

Figure 2 only shows one sample from the ' kaleidoscope'. In this spring period patterns of sudden temperature drops vary each day in strength, duration and timing, depending on the availability of nearby cold waters at depths. In spite of the variability, double temperature drops in the central basin and 

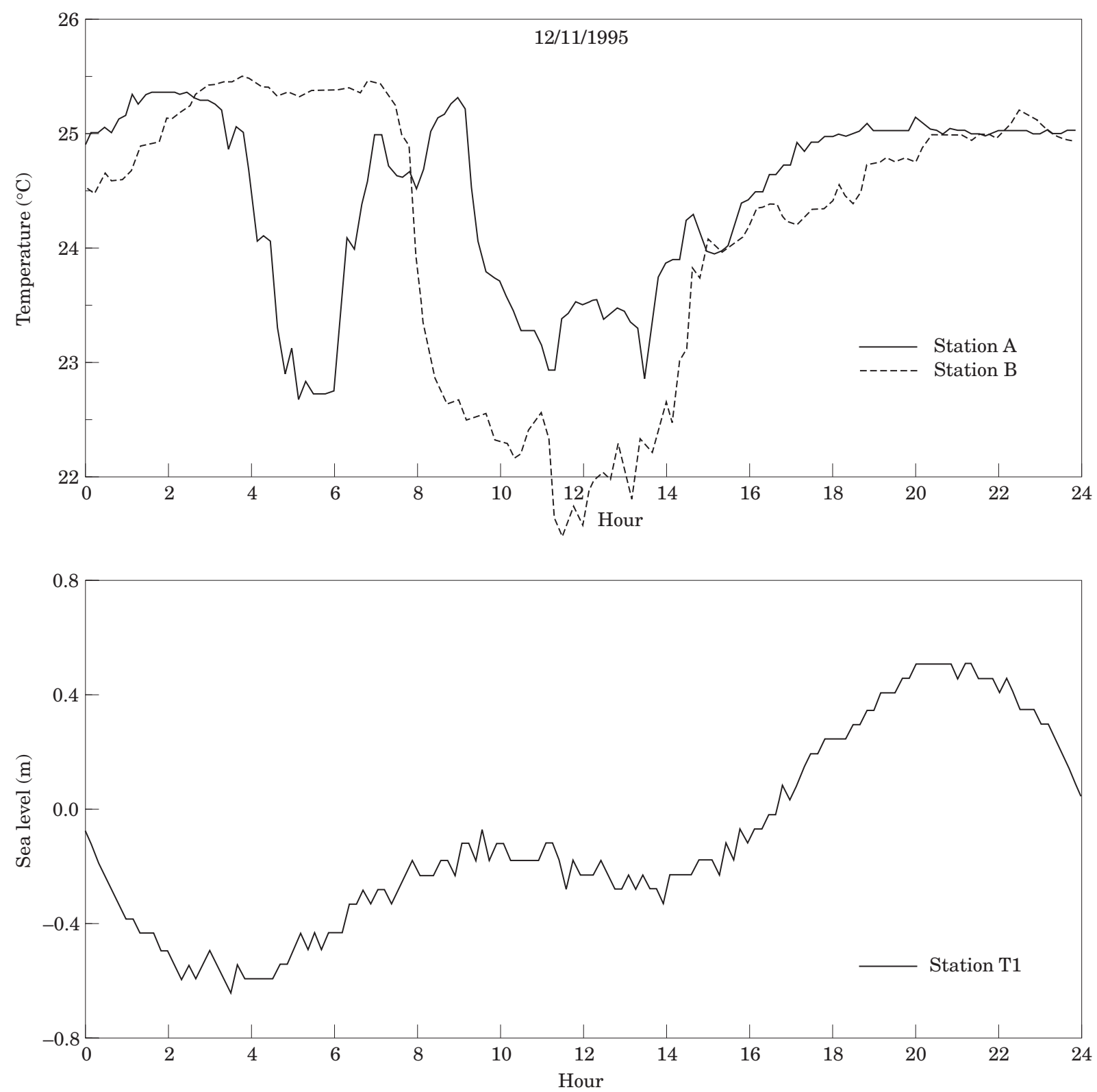

Figure 2. Temperature time series at stations A and B, and sea level at station T1 on 12 November, 1995.

single temperature drop on the east side persist daily. The degree of variability in other seasons cannot be assessed for want of better temporal coverage.

In 15-16 December, 1997, shipboard measurements of tidal currents were conducted with a shipboard ADCP (Acoustic Doppler Current Profiler) following repeated tracks as shown in Figure 3. The sea-level variation at OLB around this spring tide period (Figure 4) is typical of tropical tides. The higher high water is followed by lower low-water, lower high-water, higher low-water and higher highwater again to complete the diurnal cycle. Sealevel oscillations at other stations around the Bay are similar. To retain synopticity as much as possible, tidal flow patterns from repeated tracks are displayed in five periods of about 2-3 hours each. These five windows are shaded and denoted by Roman numerals in Figure 4.

Figure 5 shows the tidal flow patterns at $22 \mathrm{~m}$ depth in five phases. Flow patterns at other depths are similar and therefore not shown. Approaching lower low water, the prevailing ebb current in phase I period [Figure 5(a)] is essentially eastward but exhibits some clockwise deflection tendency as it flows over the continental shelf on west side of OLB. A cyclonic recirculating eddy begins to form in the northern 


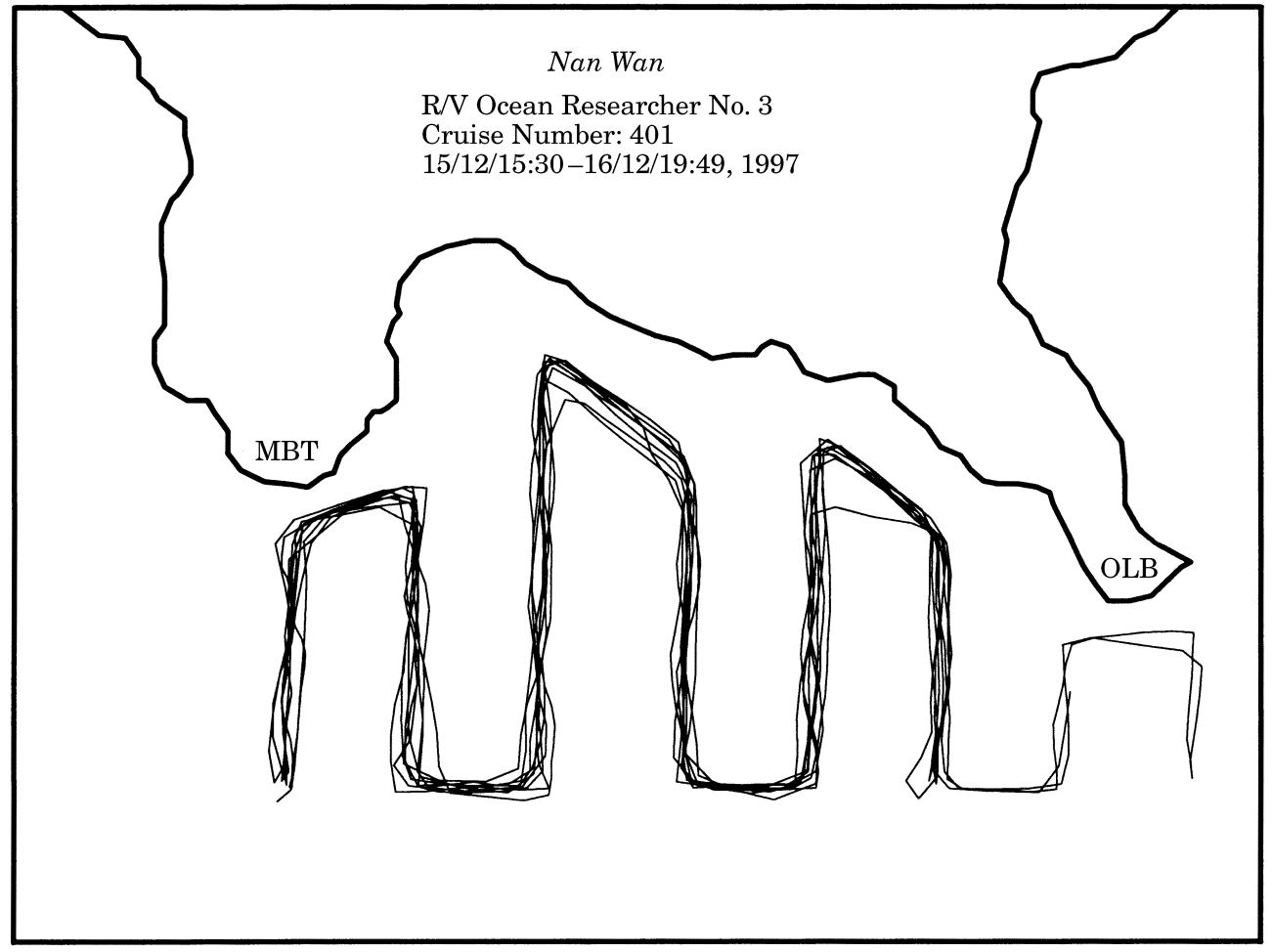

FIGURE 3. Repeated ship tracks of ADCP survey in 15-16 December, 1997.
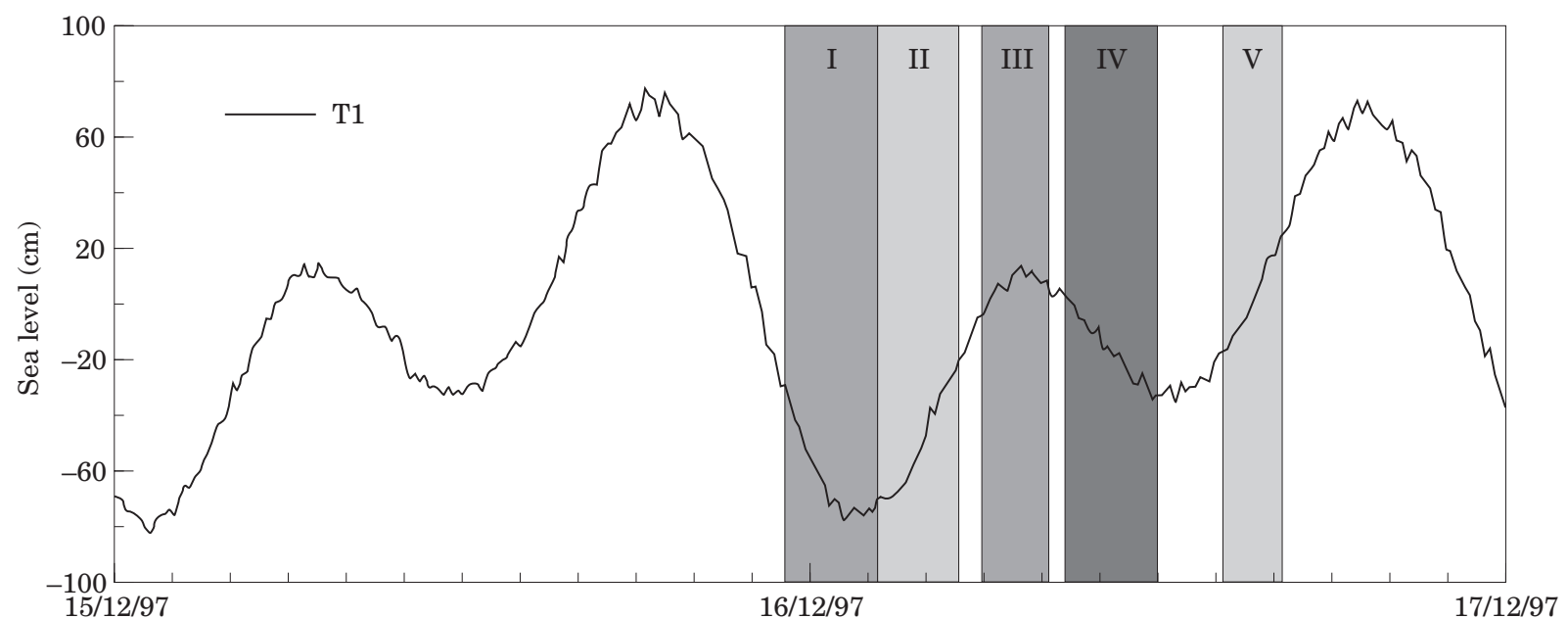

FIgURE 4. Sea-level variation at OLB or station T1 around the ADCP survey period in 15-17 December, 1997. Five shaded intervals are windows in which patterns of tidal streams are measured by ADCP.

reaches of the bay. Shortly after the lower low water, the ebbing current in phase II period [Figure 5(b)] is yet to be replaced by the flood current, pointing more offshore south of Nan Wan. The cyclonic eddy becomes well developed in the deeper reaches of the bay. Around lower high water (phase III), the westward flood current occupies the entire region [Figure 5(c)]. From lower high water to higher low water (phase IV), the prevailing tidal current [Figure 5(d)] is still westward south of the Bay. However, tidal current on east side of the Bay turns eastward, forming an anticyclonic circulation to the southwest of OLB. The anticyclone continues to expand to the entire area from phase IV to phase $\mathrm{V}$, as tidal elevation passes the higher low water and approaches higher high-water [Figure 5(e)]. 

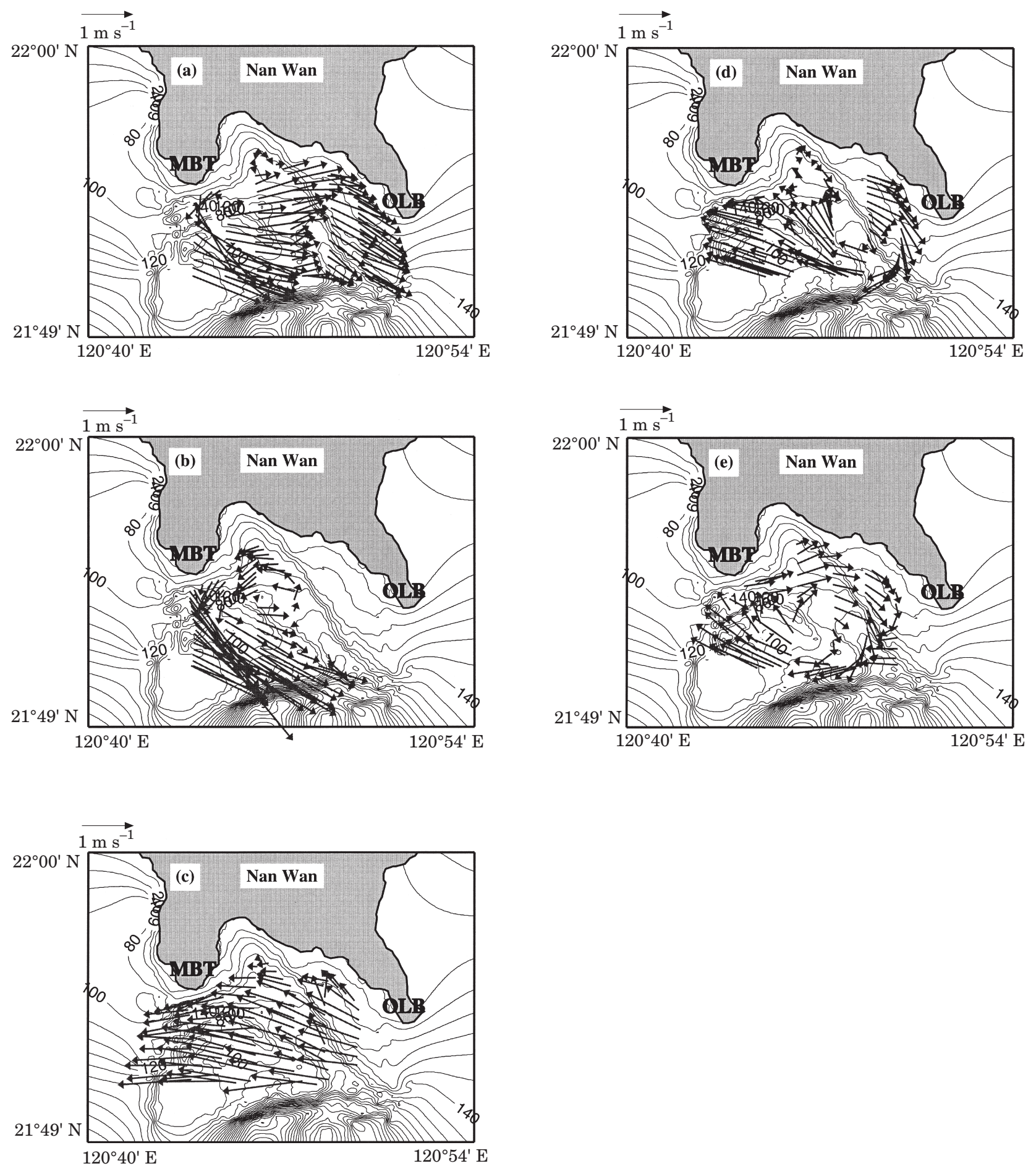

Figure 5. Tidal flow patterns at $22 \mathrm{~m}$ depth derived from ADCP survey. Panels (a, b, c, d, e) are related to tidal phases (I, II, III, IV, V) in Figure 4, respectively.

The relationship between sudden temperature drops and recirculating eddies is noted. In the central basin (station $\mathrm{A}$ ), the first temperature drop coincides with the existence of the fully developed cyclone, while the second drop is related to the developing anticyclone. Over the continental shelf on the east side (at station $\mathrm{B}$ ), the only temperature drop coincides with the developing anticyclone. Since the anticyclone first develops on the east side and expands westward in time, the anticyclone-related temperature drop is 
somewhat delayed over the central basin (at station A) with respect to that on the east side (at station $\mathrm{B}$ ).

\section{Theoretical background}

Tidal flow separation and transient eddy development behind headlands are well-known phenomena. The generating mechanism is sufficiently barotropic that these eddies can be reproduced using barotropic numerical models (Pingree \& Maddock, 1977; Tee, 1976; Awaji et al., 1980; Imasato, 1983; Geyer \& Signell, 1991). The width of these eddies is comparable to one tidal excursion length in the direction of the prevailing tide. From this simple consideration alone, one would expect a cyclonic eddy on the west side of the Bay for an eastward ebb current and an anticyclone on the east side for a westward flood current. The topography in the vicinity of Nan Wan presents complications.

In a recent numerical study (Lee et al., 1999), transient eddies in Nan Wan were examined using a three-dimensional tidal model and realistic basin bathymetry. At the time of investigation open ocean sea-level information was not available and therefore could not be used to drive realistic tidal currents. Instead, sea-level variations at any instant are assumed to be uniform along eastern and western open boundaries, both oscillating sinusoidally but with a phase difference to produce essentially zonal tidal currents south of the bay. Major results using the idealized tidal forcing are summarized below.

Tidal stream separation and return eddies behind headlands owe their existence to the inability of the prevailing tidal current to turn around promontories. Bottom topographic features deflect tidal currents and consequently may modulate the size of lee eddies. Let $f$ be the Coriolis parameter, $\zeta$ be the relative vorticity and $h$ be the local water depth. For a steady current, conservation of potential vorticity, $(f+\zeta) / h$, requires the flow to gain anticyclonic vorticity after flowing over a seamount. For oscillatory currents, the potential vorticity constraint weakens if a current oscillates with a higher frequency. Figure 6 illustrates schematically how the idealized tidal currents produce lee eddies in Nan Wan. A westward flood current flowing over the bottom ridge to the south of OLB gains anticyclonic vorticity and is expected to be deflected into the bay. This potential vorticity constraint, often demonstrated in steady-state models (Vaziri \& Boyer, 1971; Chao \& Janowitz, 1979), also applies at tidal frequencies but with a loss of efficacy. In other words, seamount-induced anticyclonic deflection decreases for tides of higher frequencies (Lee et al., 1999). The bayward deflection suppresses the would-be expan-
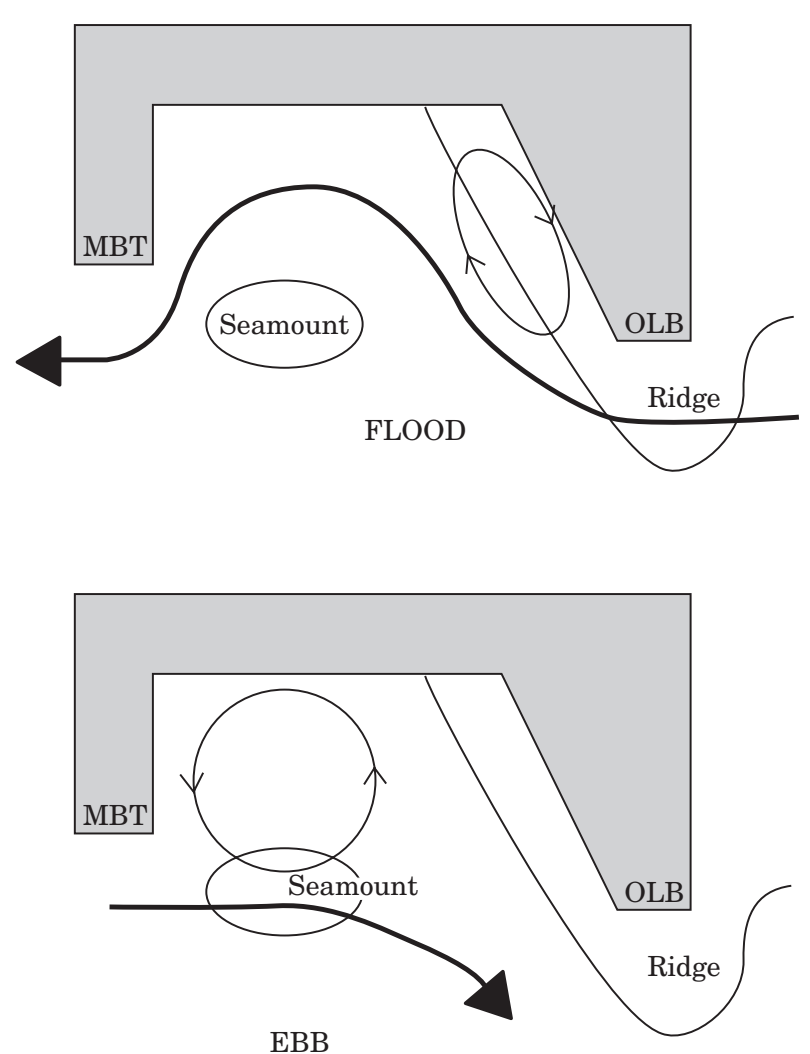

FIGURE 6. A schematic illustration of prevailing tidal streams and recirculation eddies during flood and ebb periods inferred from a protocol tidal model. Assuming tidal streams are essentially zonal south of the Bay, the cyclonic eddy grows during an ebb but the anticyclonic eddy during a flood is confined to the east side. The rapid expansion of the anticyclonic eddy as revealed by recent observations [Figure 5(d and e)] was not captured by this earlier model.

sion of the anticyclonic lee eddy and confines it to mostly over the continental shelf on the east side. For an eastward ebb current, the cyclonic eddy in the bay is primarily caused by the zonally elongated seamount at the bay mouth. Flowing over the seamount, the eastward current is deflected southward following the potential vorticity constraint, and the cyclonic eddy is enlarged in the process. If the seamount is removed, the cyclone will be reduced in strength and size considerably (Lee et al., 1999). In summary, the protocol tidal model of Lee et al. (1999) captured the generating mechanism of cyclones but failed to produce the rapid expansion of anticyclones. The latter was not revealed until the recent ADCP survey (Figure 5). The protocol model thus suggested that the upwelling is a once-per-day event in deeper (central and west) part of the basin.

Two interrelated discrepancies remain to be resolved. One is the rapid expansion of the anticyclone during floods. The other is the twice-per-day 


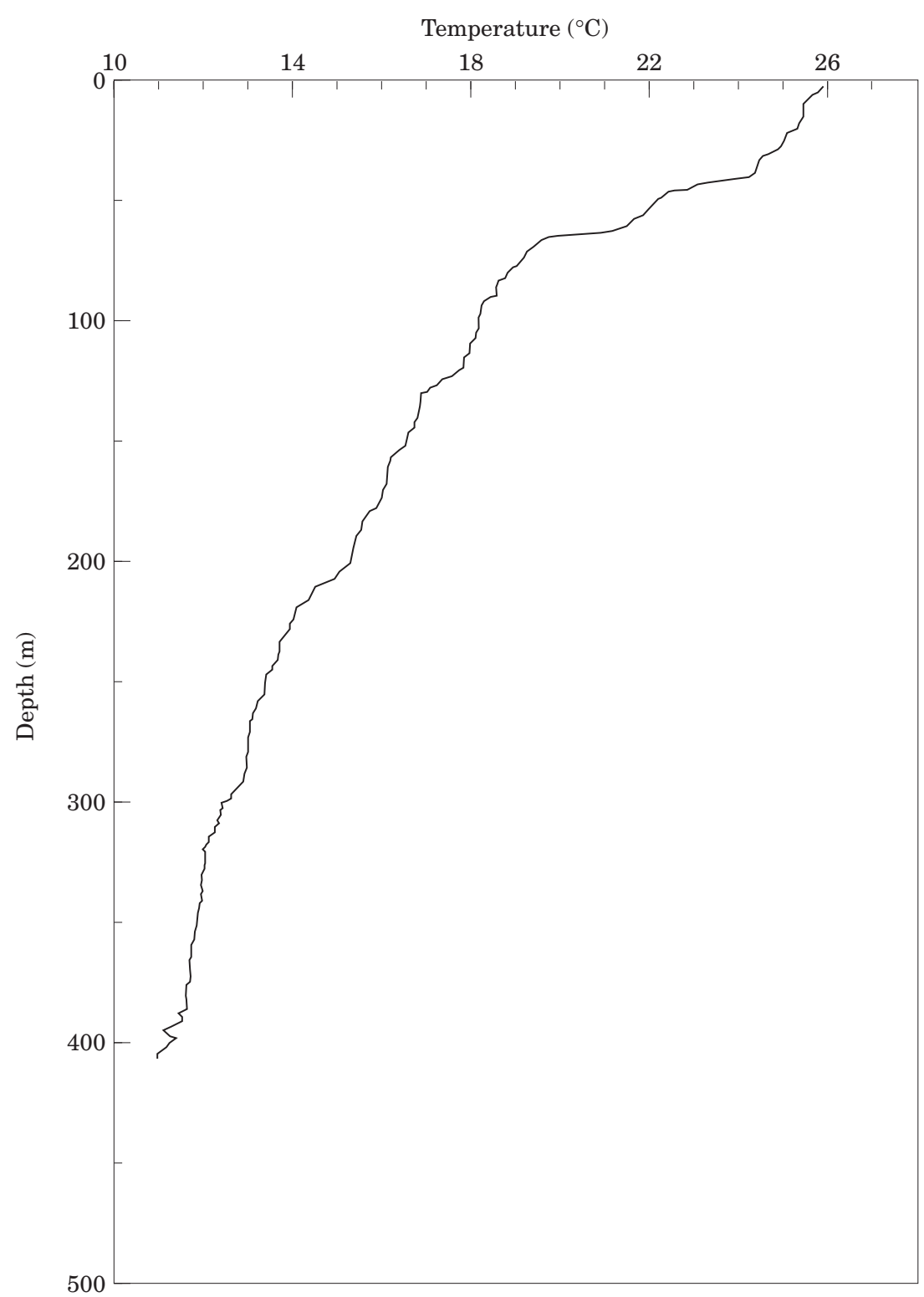

FIGURE 7. A characteristic temperature profile at station $\mathrm{X}\left(21^{\circ} 51^{\prime} \mathrm{N}, 120^{\circ} 48^{\prime} \mathrm{E}\right)$ on 18 October, 1995 . See Figure 1 for the location.

occurrence of upwelling in central and western parts of the basin. The remaining of this paper seeks to resolve these issues by incorporating previously unavailable and realistic tidal forcing into the model.

\section{Model}

A three-dimensional model similar to Lee et al. (1999) is used below. The model is formulated under hydrostatic and Boussinesq approximations. The numerical code is based on the Bryan-Cox model except the rigid-lid assumption is relaxed to accommodate for a free surface. Details of model formulation were given in Chao et al. (1996). The initial stratification varies only in the vertical direction, given by the typically observed temperature profile in Nan Wan (Figure 7). The observed salinity varies little with depths; salinity in the model is therefore set to the commonly observed value of 34.6 (using the Practical Salinity Scale). The model ocean is initially motionless and subsequently driven by sea-level oscillations on open boundaries.

Four tide gauge stations (T1, T2, T3, T4 in Figure 1) were maintained from 17 October to 23 November to measure sea-level fluctuations at five-minute intervals. Straight lines joining T1, T2, T3 and T4 
TABle 1. Periods ( $T_{O}$ in hours), amplitudes $\left(A_{O}\right.$ in $\left.\mathrm{cm}\right)$ and time lags $(\Theta$ in hours $)$ of dominant tidal harmonic components at stations $\mathrm{T} 1, \mathrm{~T} 2, \mathrm{~T} 3$ and $\mathrm{T} 4$ in Figure 1. Time lags are relative to 11/06/17:00, 1995 GMT

\begin{tabular}{|c|c|c|c|c|c|c|c|c|c|}
\hline \multirow[b]{2}{*}{ Tides } & \multirow[b]{2}{*}{$\mathrm{T}_{\mathrm{o}}$} & \multicolumn{2}{|c|}{$\mathrm{T} 1$} & \multicolumn{2}{|c|}{$\mathrm{T} 2$} & \multicolumn{2}{|c|}{ T3 } & \multicolumn{2}{|c|}{$\mathrm{T} 4$} \\
\hline & & $A_{o}$ & $\Theta$ & $\mathrm{A}_{\mathrm{o}}$ & $\Theta$ & $\mathrm{A}_{\mathrm{O}}$ & $\Theta$ & $\mathrm{A}_{\mathrm{o}}$ & $\Theta$ \\
\hline $\mathrm{Q}_{1}$ & $26 \cdot 87$ & $4 \cdot 77$ & $14 \cdot 13$ & $4 \cdot 65$ & $15 \cdot 79$ & 3.95 & $15 \cdot 97$ & $3 \cdot 76$ & $17 \cdot 43$ \\
\hline $\mathrm{O}_{1}$ & $25 \cdot 82$ & $19 \cdot 4$ & $14 \cdot 92$ & $19 \cdot 06$ & $16 \cdot 09$ & $19 \cdot 81$ & $16 \cdot 33$ & $20 \cdot 33$ & $16 \cdot 94$ \\
\hline $\mathrm{K}_{1}$ & 23.93 & $22 \cdot 8$ & $17 \cdot 35$ & $15 \cdot 88$ & $18 \cdot 09$ & $21 \cdot 58$ & $16 \cdot 40$ & $22 \cdot 27$ & $16 \cdot 83$ \\
\hline $\mathrm{N}_{2}$ & $12 \cdot 66$ & $7 \cdot 63$ & $5 \cdot 92$ & $5 \cdot 69$ & $5 \cdot 14$ & $4 \cdot 6$ & $6 \cdot 38$ & $4 \cdot 69$ & $7 \cdot 12$ \\
\hline $\mathrm{M}_{2}$ & $12 \cdot 42$ & $28 \cdot 43$ & $6 \cdot 23$ & $25 \cdot 21$ & $6 \cdot 09$ & $23 \cdot 18$ & $6 \cdot 54$ & $24 \cdot 79$ & $7 \cdot 56$ \\
\hline $\mathrm{S}_{2}$ & $12 \cdot 0$ & $12 \cdot 29$ & $7 \cdot 47$ & $14 \cdot 4$ & $6 \cdot 90$ & $10 \cdot 86$ & $6 \cdot 73$ & $11 \cdot 94$ & $7 \cdot 88$ \\
\hline
\end{tabular}

delineate the boundaries separating Nan Wan and vicinity from adjacent seas. Station T2 was unfortunately lost during the period but was reestablished from 22 August to 22 September, 1996. Table 1 shows the period (in hours), amplitude (in $\mathrm{cm}$ ) and phase (in hours) of six major harmonic components at the four stations. These semi-diurnal and diurnal tidal harmonics resolve most of the sea-level variations including a pronounced spring-neap cycle. Sea-level variations along open ocean boundaries are determined from Table 1 by linearly interpolating and extrapolating tidal characteristics among the four stations. The model starts from a time when tides enter a typical spring period.

Leaving sea level aside, other boundary conditions follow. Let $(\mathrm{u}, \mathrm{v}, \mathrm{w})$ be positive eastward, northward and upward, respectively. Horizontal velocities on open boundaries are extrapolated from the model interior, so that

$$
\partial(u, v) / \partial n=0
$$

where $n$ is normal to a boundary. The vertical velocity is set to zero on open boundaries. Temperatures $(T)$ on open boundaries are advected by the flow normal to the boundary $\left(u_{n}\right)$, so that

$$
\partial T / \partial t+u_{n} \partial T / \partial n=0
$$

In case of inflow, inflow temperature is set to the characteristic temperature as shown in Figure 7. Land boundaries are impenetrable, impermeable and no-slip. At the sea surface, no momentum or heat flux is imposed. At the ocean bottom, there is no normal flow or heat flux and a quadratic bottom stress is imposed with a dimensionless drag coefficient of $0 \cdot 0025$.

The model resolution is $200 \mathrm{~m}$ in horizontal directions and $7.5 \mathrm{~m}$ in the vertical. As indicated in
Figure 1, the zonal extent of model domain is wider than the Bay. Northern open boundaries to the east and west of the Bay are truncated by zonal walls. Horizontal mixing is Laplacian. In the model interior the mixing coefficient is $7 \times 10^{4} \mathrm{~cm}^{2} \mathrm{~s}^{-1}$ for momentum and $7 \times 10^{3} \mathrm{~cm}^{2} \mathrm{~s}^{-1}$ for temperature. To suppress computation instability, sponge layers as indicated in Figure 1 are used near the three open boundaries. The horizontal mixing coefficient for momentum increases linearly up to 20 times of their nominal values toward open boundaries inside sponge layers. Viscosity and diffusion in the vertical are parameterized following Pacanowski and Philander (1981). The model resolves barotropic and baroclinic components with different time steps using the standard mode-splitting technique (Simons, 1974). The time step is $0.3 \mathrm{~s}$ for the barotropic mode and $12 \mathrm{~s}$ for the baroclinic model. The Coriolis parameter is set to $5 \cdot 448 \times 10^{-5} \mathrm{~s}^{-1}$.

East and west open boundaries are close to the headlands to take advantage of the availability of tide gage data. In the numerical experiments to be discussed below, land masses to the east and west of the bay are stretched out zonally to intersect the open boundaries at the right angle. If computationally stable, solutions are visually indistinguishable whether the land masses are stretched or not. Occasional instability results if landmasses are not stretched out zonally.

Starting from a motionless state, the initial transient decays and the circulation enters quasi-cyclical equilibrium after about two diurnal cycles. The fourth cycle is discussed below. The quasi-cyclical solution shows a weak and slow drift from one diurnal cycle to the next. The drift is caused partly by intertidal sea-level variations and partly by the slowly varying stratification in the basin. Lacking heat input from the sea surface, the ocean stratification weakens gradually and the solution is becoming more barotropic over 
long time scales. To establish a steady state requires knowledge of heat flux at the air-sea interface, which has never been measured in our observation efforts. Leaving weak intertidal variations aside, the solution is able to reproduce essential features revealed from observations.

\section{Numerical results}

Figure 8 shows snapshots of surface tidal currents (left panels) and temperature fields at $26.25 \mathrm{~m}$ depth (right panels) at four-hour intervals in the fourth diurnal cycle. Sea level in relation to the full diurnal cycle is shown on top of OLB in each panel. Top panels [Figure 8(a and g)] are around lower low-water; the eastward tidal stream follows the curvature of the bay to turn anticyclonically. A small cyclonic eddy begins to form in the northern extremity of the bay. The overall flow pattern resembles the observed tidal flow in phase I period [Figure 5(a)]. A cold pool on the eastern half of the basin [Figure $8(\mathrm{~g})$ ] is the remnant of cold waters upwelled at the end of previous tidal cycle.

From lower low-water to lower high-water, Figure 8 (b) and (c) shows the expansion and intensification of cyclonic eddy at the expense of the eastward tidal stream. Shortly after lower high water, Figure 8(c) resembles the observed tidal current in phase II period [Figure 5(b)]: a well developed cyclonic eddy in the bay and a southeastward tidal stream to the south. Corresponding temperature fields in Figure 8(h) and (i) indicates the close relation between the cold anomaly and cyclonic eddy. As the cyclone grows and intensifies, cold waters are upwelled from below. Sea surface topography (not shown) corresponding to Figure 8 (b) and (c) is generally higher to the east. Conceivably, the inertia maintains the eastward tidal stream south of the bay against the pressure gradient force. Westward flow at the coast is aided by the pressure gradient.

Shortly after the higher low-water, the eastward flood current enters the bay, following the curvature of the bay to turn cyclonically [Figure $8(d)$ ]. The flow pattern bears some resemblance to the observed tidal flow in phase III period [Figure 5(c)]. At this time the cyclone-induced cold anomaly is mostly dispersed [Figure 8(j)]. Cold anomalies begin to appear south and west of OLB, apparently induced by orographic lifting of flood current flowing over the bottom ridge south of OLB.

Around higher high water, Figure 8(e) and (f) captures the rapid south-westward expansion of the anticyclone from west of OLB to the entire Bay in 4 hours. South of the anticyclone is the prevailing westward flood current. The rapid expansion of anticyclonic eddy was revealed earlier by the ADCP survey during phase IV and phase $\mathrm{V}$ periods [Figure 5(d-e)]. Concurrent temperature fields [Figures $8(\mathrm{k}$ and 1)] show the anticyclone-induced upwelling of cold waters from below. The cold pool expands with the expanding anticyclone.

To better quantify the model-data comparison, Figure 9 shows the model-produced temperature time series at stations A and B (Figure 1) for the fourth diurnal cycle. The lower panel of Figure 9 shows the observed sea-level variation at tide station $\mathrm{T} 1$ and its approximation by six major harmonics; the latter was used as part of the boundary forcing to drive the model. At station A, two temperature drops of the day are prominent. The first drop coincides with the occurrence of a fully developed cyclone, while the second occurs as the anticyclone is expanding rapidly. There is only one sizeable temperature drop at station $B$, occurring before the second temperature drop at station A. The only drop at station B begins at the early expansion phase of the anticyclone.

While the overall agreement with observed temperature drops (Figure 2) is apparent, discrepancies remain. The model-produced temperature drops are weaker than observed ones. As commented on earlier, stratification in the model basin weakens slowly in time. The stratification must be maintained by realistic heat input from the air-sea interface, which is not available at this time. In Figure 8, the rapid expansion phase of the anticyclonic eddy is delayed by a few hours compared with observations in Figure 5. In consequence, the anticyclone-induced temperature drops (second one at station $\mathrm{A}$ and the only one at station B) are similarly delayed. The tidal model is driven by boundary forcing derived from observed tides near four corners of the model domain; mesoscale features are likely under-represented in this practice. Open-ocean boundary forcing can be improved in the future by employing more tide gauges along boundaries, or by obtaining sea-level variations from a credible large-scale tidal model. Neither option is available at this time.

Tide-induced residual surface circulation around springs (Figure 10) is derived from the fourth tidal cycle. The residual circulation consists of an expansive anticyclone on the east side and to the south of the bay and an incomplete cyclonic circulation in western reaches of the Bay. Since the transient cyclone and anticyclone in Figures 5 and 8 do not exist in the same area, both are captured after time averaging. The residual currents are rather strong because prevailing flood and ebb currents do not follow the same path. 

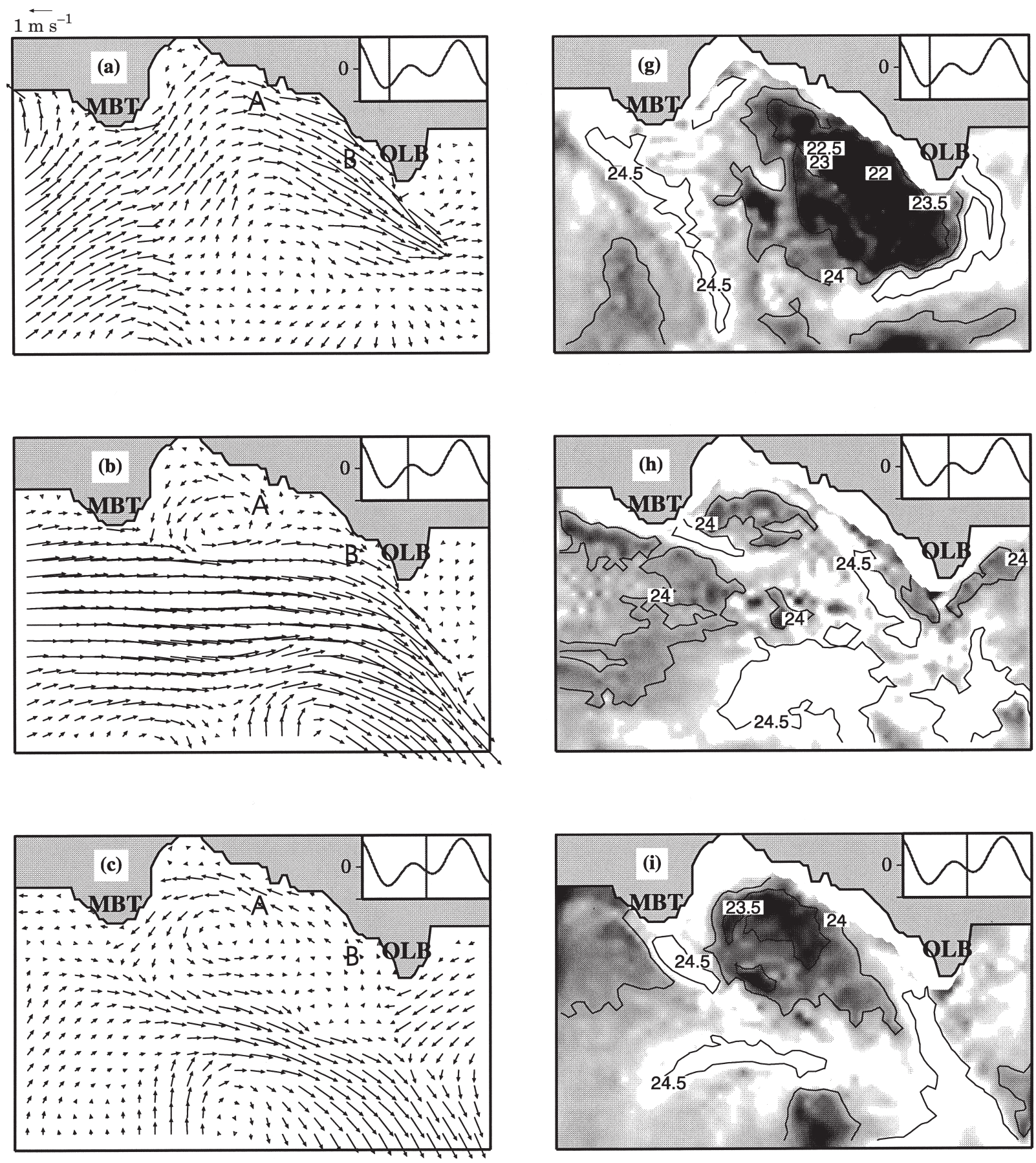

FIGURE 8. Model-produced surface tidal currents (left panels) and temperature fields at $26 \cdot 25 \mathrm{~m}$ depth (right panels) at four-hour intervals in the fourth diurnal cycle. Sea level in relation to the full diurnal cycle is shown on top of OLB in each panel. Stations A and B are marked on left panels.

\section{Discussion and conclusions}

Around spring, the two tides of the day in Nan Wan shows a maximum in diurnal inequality where a large tide and a half tide alternate. The so-called 'tropical tides ' induce a cyclonic recirculating eddy on the west side of the basin shortly after lower low-water, and an anticyclone rapidly expanding from the east side before higher high-water. Both eddies induce upwelling of cold waters from below. The size disparity between cyclonic and anticyclonic eddies affect the characteristics of sudden temperature drops. In central and 

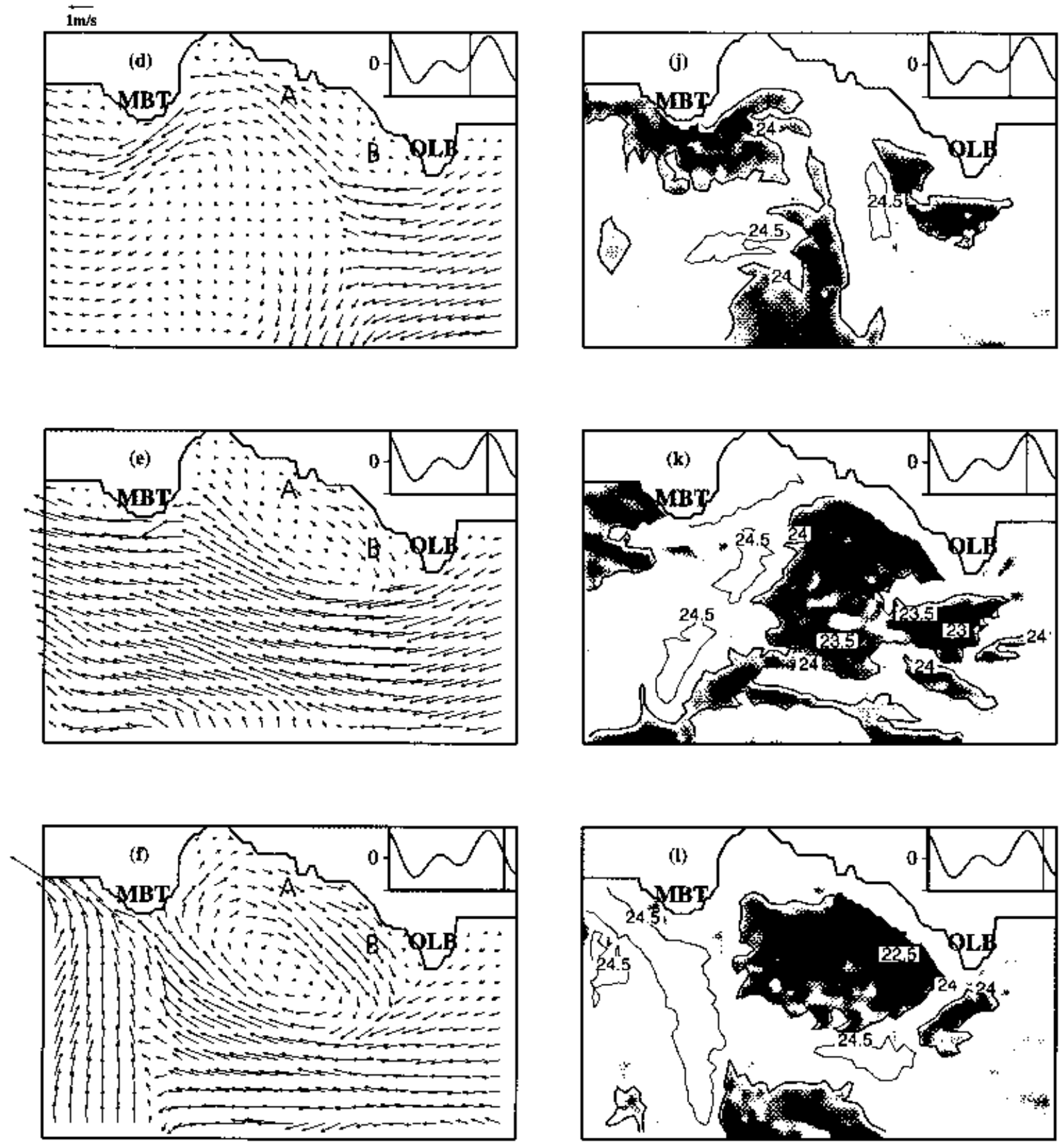

Figure 8. Continued.

western parts of the basin, both cyclone- and anticyclone-induced upwelling events are felt and there are two temperature drops in a typical day around springs. On the east side, only the anticycloneinduced upwelling is felt and the temperature drop is once per day.

Model sensitivity to tidal phases imposed along open-ocean boundaries should be emphasized. In an earlier model (Lee et al., 1999), sea-level variations were assumed to be uniform along eastern and western ocean boundaries; flood and ebb currents thus generated became more zonal south of the bay. As illustrated in Figure 6, the scenario run leads to a recirculating anticyclonic eddy confined over the continental shelf on the east side, unable to expand to the entire basin. With more realistic tidal phases imposed herein, the westward flood current south of OLB contains a sizeable southward component [Figure 8 (d, 

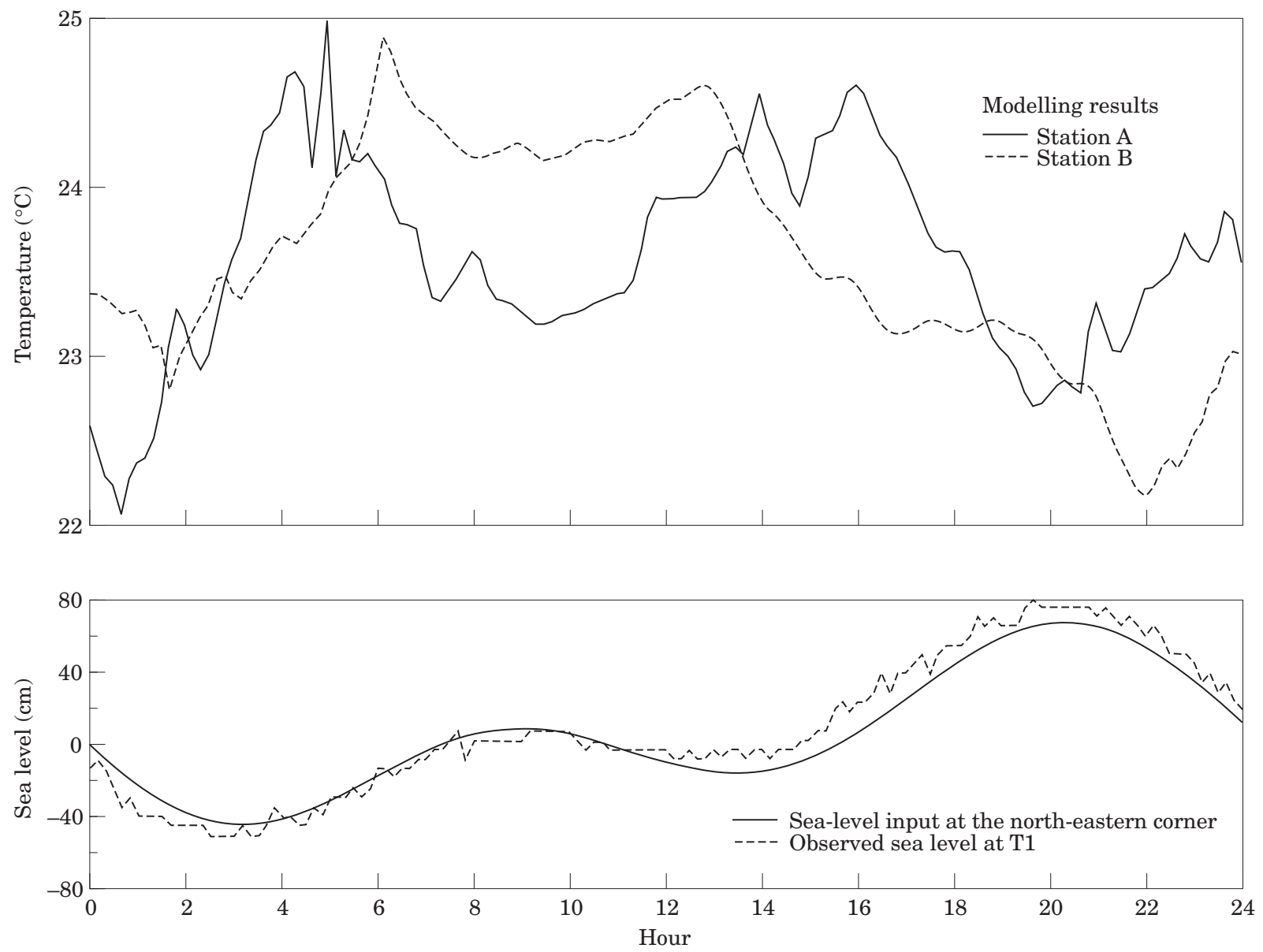

FIGURE 9. Model-produced temperature time series at stations A and B (top panel) in the fourth diurnal cycle. The observed sea-level variation at station $\mathrm{T} 1$ and its approximation by six tidal harmonics are shown by dashed and solid lines, respectively, in the bottom panel.

e and f)]. More waters are drawn out of the bay by this flood current; the semi-enclosed basin becomes a low pressure center supporting the rapid expansion of the anticyclonic eddy.

It should also be emphasized that the centrifugal force dominates over Coriolis force for both cyclonic and anticyclonic eddies. In consequence, both eddies induce upwelling. Let $V_{O}$ be a characteristic swirling speed and $r_{0}$ be the characteristic radius. The ratio of centrifugal acceleration $\left(V_{0}^{2} / r_{0}\right)$ to Coriolis acceleration $\left(f V_{0}\right)$ is a Rossby number, $R=V_{o} / f r_{0}$. Observed eddies in Figure 5 can be used to estimate $R$. For the cyclonic eddy in Figure 5(b), $r_{0}$ is about $2 \mathrm{~km}$ and $V_{0}$ is about $0.5 \mathrm{~m} \mathrm{~s}^{-1}$, yielding $R \approx 4.5$. For the expanding anticyclone in Figure $5(\mathrm{~d}), r_{0}$ is about $2 \cdot 8 \mathrm{~km}$ and $V_{O}$ is about $1 \mathrm{~m} \mathrm{~s}^{-1}$, yielding $R \approx 6 \cdot 7$. The centrifugal force leads to eddy expansion and upwelling.

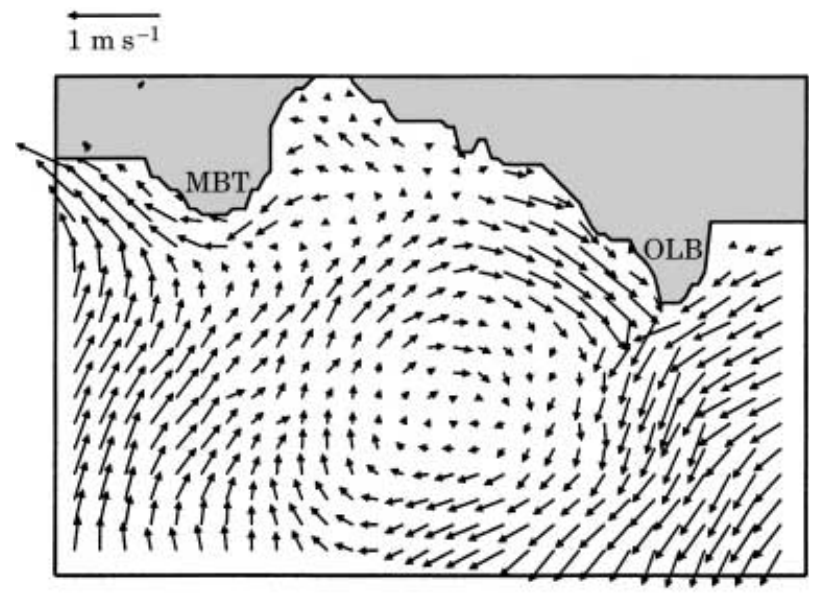

FIGURE 10. Tide-induced residual circulation around spring tidal periods in Nan Wan, derived by time-averaging of model-produced surface tidal currents over the fourth diurnal cycle. 


\section{Acknowledgements}

Authors HJL and KLF were supported by the National Research Council of the Republic of China under grant NSC 88-2611-M-002-002. Author KTY was supported by Taiwan Ocean Technology Consulting Company. Author SYC was supported by the U.S. National Science Foundation under grant OCE-95-04959. Field works were assisted by Yung-Jing Tsau, Tsong-Teh Su, Chao-Chang Lin and Jang-Ming Chiou.

\section{References}

Awaji, T. A., Imasato, N. \& Kunishi, H. 1980 Tidal exchange through a strait: A numerical experiment using a simple model basin. Fournal of Physical Oceanography 10, 1499-1508.

Chao, S.-Y. \& Janowitz, G. S. 1979 The effect of a localized topographic irregularity on the flow of a boundary current along the continental margin. Fournal of Physical Oceanography 9, 900-910.

Chao, S.-Y., Shaw, P.-T. \& Wu, S. Y. 1996 El Niño modulation of the South China Sea circulation. Progress in Oceanography 38, 51-93.
Geyer, W. R. \& Signell, R. 1991 Measurement and modeling of the spatial structure of nonlinear tidal flow around a headland. In Tidal Hydrodynamics (Parker, B. B., ed.). John Wiley and Sons, New York, pp. 403-418.

Imasato, N. 1983 What is tide-induced residuals current? fournal of Physical Oceanography 13, 1307-1317.

Lee, H.-J., Chao, S.-Y., Fan, K.-L., Wang, Y.-H. \& Liang, N.-K. 1997 Tidally induced upwelling in a semi-enclosed basin: Nan Wan Bay. Fournal of Oceanography 53, 467-480.

Lee, H.-J., Chao, S.-Y. \& Fan, K.-L. 1999 Flood-ebb disparity of tidally induced recirculating eddies in a semi-enclosed basin: Nan Wan Bay. Continental Shelf Research 19, 871-890.

Pacanowski, R. C. \& Philander, S. G. H. 1981 Parameterization of vertical mixing in numerical models of tropical oceans. Fournal of Physical Oceanography 11, 1443-1451.

Pingree, R. D. \& Maddock, L. 1977 Tidal eddies and coastal discharge. Fournal of Marine Biology Association of United Kingdom 57, 869-875.

Simons, T. J. 1974 Verification of numerical models of Lake Ontario: Part I. Circulation in spring and early summer. Foumal of Physical Oceanography 4, 507-523.

Tee, K. T. 1976 Tide-induced residual current, a 2-D nonlinear numerical tidal model. Fournal of Marine Research 34, 603-628.

Vaziri, A. \& Boyer, D. L. 1971 Rotating flow over shallow topographies. Fournal of Fluid Mechanics 50, 79-95. 
